\title{
Área de estructuras respiratorias y su efecto en la regulación del equilibrio ácido-base en dos especies de cangrejos porcelánidos intermareales, Petrolisthes laevigatus y Petrolisthes violaceus
}

\author{
Area of respiratory structures and their effect on acid-base balance regulation in two species \\ of porcelain intertidal crabs, Petrolisthes laevigatus and Petrolisthes violaceus
}

\author{
Moisés A. Vargas ${ }^{1}$, Marcelo E. Lagos ${ }^{1}$, Daniela A. Contreras ${ }^{1}$ y Cristian W. Cáceres ${ }^{1}$ \\ ${ }^{1}$ Departamento de Ecología Costera, Facultad de Ciencias, Universidad Católica de la Santísima Concepción, Casilla 297, \\ Concepción, Chile. ccaceres@ucsc.cl
}

\begin{abstract}
Porcelain crabs that habiting intertidal zone are continuously exposed to desiccation conditions for which they have developed specific physiological and morphological adaptations. In this study, the relationship between gill surface area and walking legs membranes, and acid-base regulation of two species of intertidal crabs (Petrolisthes) were measured and compared. Petrolisthes laevigatus inhabits on the high intertidal zone, while $P$. violaceus is on the lower intertidal zone. Both species have the same surface gill area, but $P$. laevigatus has a bigger area on their walking legs membranes in comparison to $P$. violaceus. The difference in membrane area may be relevant in their adaptation to air exposure. We found differences on their acid-base balance between these two species and between individuals with and without functional membranes. Petrolisthes laevigatus would have a higher level of homeostatic regulation in the emersion periods in comparison to $P$. violaceus.
\end{abstract}

Key words: Gas windows, crustaceans, intertidal, respiration, emersion

Resumen.- Los crustáceos porcelánidos intermareales están continuamente expuestos a condiciones de desecación, por lo que han desarrollado adaptaciones fisiológicas y morfológicas específicas. En este estudio se midió y comparó el área total branquial, las membranas respiratorias y la capacidad de regular el equilibrio ácido base en exposición aérea con las membranas obstruidas y libres de dos especies de jaibas del género Petrolisthes. Petrolisthes laevigatus habita en la zona alta del intermareal mientras que $P$. violaceus habita en la zona baja. Ambas especies presentaron la misma área branquial, pero $P$. laevigatus presentó una mayor área de la membrana de los apéndices locomotores en comparación a $P$. violaceus. La diferencias del área de la membrana pueden ser relevantes para la adaptación a la exposición aérea, ya que se encontraron diferencias en el equilibrio ácido-base entre especies y entre individuos con membranas funcionales y membranas obstruidas. $P$. laevigatus tendría un mayor grado de regulación homeostática lo que le daría ventajas fisiológicas para soportar la emersión en comparación a $P$. violaceus.

Palabras clave: Membranas respiratorias, crustáceos, intermareal, respiración, emersión

\section{INTRODUCCIÓN}

La transición de los organismos acuáticos a sistemas terrestres, es uno de los hitos más relevantes en la historia evolutiva de los animales. Este proceso implica demandas ecológicas, anatómicas y fisiológicas que solo ciertos grupos, entre los cuales destacan algunos crustáceos decápodos, han sido capaces de enfrentar exitosamente (Greenaway 2003).

En estudios comparativos en crustáceos, se ha observado que la superficie primaria de intercambio gaseoso, el área superficial de las branquias, disminuye a medida que aumenta el grado de independencia de la especie a sistemas acuáticos (Hawkins \& Jones 1982, Greenaway 1984, Farrelly \& Greenaway 1987). En sistemas marinos, los crustáceos poseen largas branquias con lamelas empaquetadas que se ubican en cámaras en ambos lados del cefalotórax. Se ha descrito que las branquias que ocupan la porción anterior de cada cámara tienen primariamente una función de intercambio gaseoso y las branquias posteriores participan activamente en los procesos de osmoregulación (Farrelly \& Greenaway 1994, Onken et al. 2003). Por otra 
parte, en sistemas terrestres las branquias en los crustáceos se encuentran reducidas en número y tamaño, presentando lamelas quitinosas que permanecen rígidas, impidiendo su colapso, facilitando el intercambio gaseoso aéreo en las cámaras branquiales (Hawkins \& Jones 1982, Farrelly \& Greenaway 1994).

En crustáceos acuáticos la exposición al aire provoca una retención del $\mathrm{CO}_{2}$ en la hemolinfa, con la consecuente acidificación (Varley \& Greenaway 1992, Luquet \& Ansaldo 1997). Este cambio de $\mathrm{pH}$ activa mecanismos homeostáticos para la regulación del equilibrio acido base como el intercambio de iones a nivel branquial, principalmente $\mathrm{Cl}^{-}$por $\mathrm{HCO}_{3}{ }^{-}, \mathrm{Na}^{+}$por $\mathrm{H}^{+}$y la liberación de $\mathrm{Ca}^{2+}$ del exosqueleto, que facilitan la excreción de $\mathrm{CO}_{2}$ y la regulación del pH de la hemolinfa (Henry \& Wheatly 1992, Wheatly \& Henry 1992, Lagos \& Cáceres 2008).

Existen crustáceos capaces de mantener una respiración bimodal, que es la capacidad de realizar intercambio gaseoso en agua y aire (Hawkins et al. 1982, Innes et al. 1986, Maitland 1990, Henry 1994, Luquet et al. 1998); este tipo de respiración es efectuado principalmente en otros sitios de intercambio gaseoso, los cuales pueden ser una fina cutícula altamente vascularizada en la superficie del cefalotórax, al costado de la cámara branquial (Henry 1994, Halperin et al. 2000, Greenaway 2003) o segmentos descalcificados en la cara anterior de los apéndices locomotores denominados membranas respiratorias, las cuales les permitirían soportar largos periodos de exposición al aire (Stillman \& Somero 1996, Stillman 2000).

Petrolisthes laevigatus (Guerin, 1835) y Petrolisthes violaceus (Guerin, 1831) son dos especies de porcelánidos comunes en las costas rocosas de Chile, ambas se distribuyen desde el norte del Perú hasta Aysén, Chile. Estas especies se encuentran bajo rocas ("bolones") y usualmente presentan un patrón de zonación discreto, $P$. laevigatus ocupa la zona media y superior del intermareal y $P$. violaceus se encuentran en la zona media baja del intermareal y en el sub-mareal somero (Viviani 1969).

Dada su distribución vertical y el régimen de mareas, estos organismos están rutinariamente expuestos a diferentes periodos de exposición aérea. La especie del intermareal alto se ve expuesta a dos periodos de emersión diarios, mientras que para la especie del intermareal bajo su exposición es menor, dependiendo de la altura de las mareas (Stillman \& Somero 1996). Dado lo anterior, se espera que las membranas de los apéndices locomotores sean capaces de suplir, al menos en parte, el rol de las branquias, facilitando la regulación del equilibrio ácido base durante la exposición aérea. El objetivo de este trabajo es evaluar el efecto de estas membranas en la mantención del equilibrio ácido base en Petrolisthes laevigatus y $P$. violaceus durante la emersión. Para esto, se determinó el área de las membranas, los cambios de $\mathrm{pH}$ y concentraciones de $\mathrm{HCO}_{3}{ }^{-}$y $\mathrm{PCO}_{2}$ en la hemolinfa de ambos Petrolisthes, en membranas funcionales y no funcionales a diferentes tiempos de exposición aérea.

\section{MATERIAL Y MÉTODOS}

Los individuos de Petrolisthes laevigatus y $P$. violaceus ( $\mathrm{N}=175$ por especie), fueron recolectados manualmente en la zona intermareal de Lenga, Bahía San Vicente, Chile $\left(36^{\circ} 45^{\prime} \mathrm{S}-70^{\circ} 10^{\prime} \mathrm{W}\right)$ y trasladados al laboratorio de Fisiología Animal de la Universidad Católica de la Santísima Concepción. Cada ejemplar fue pesado con una balanza analítica de 0,005 g de sensibilidad y posteriormente aclimatados en recipientes con agua de mar circulante por tres días a $13^{\circ} \mathrm{C}$ y mantenidos sin alimento (Chen \& Chia 1997, Lucu \& Devescovi 1999, Castillo et al. 2009).

\section{ÁrEA DE ESTRUCTURAS RESPIRATORIAS}

Para medir el área de la superficie respiratoria branquial, se disectó cada branquia de la cámara branquial derecha, midiendo su longitud y cuantificando el número total de lamelas presentes; posteriormente se removió una lamela de posición central (Hawkins \& Jones 1982), la cual fue fotografiada bajo lupa binocular con una cámara digital Motic $^{\circledR}$. El área total de cada lamela fue obtenida con el software Motic Image Plus 2.0 ML. Para estimar el área total branquial, se multiplicó el valor obtenido por el número total de lamelas de cada branquia y por el número total de branquias presentes en ambas cámaras branquiales (Hawkins \& Jones 1982, Astall et al. 1997).

Para medir el área total de las membranas de los apéndices locomotores (membranas respiratorias), se realizó la disección longitudinal de cada pereiópodo del lado derecho, separando del mesopodito la cara anterior de la posterior, extrayendo la musculatura presente. De igual modo se determinó el área de cada membrana.

\section{EQUiLIBRIo ÁCIDO-BASE}

Para evaluar los mecanismos de regulación ácido-base durante períodos de emersión, los individuos de cada especie fueron asignados a diferentes tiempos de exposición aérea, 0 (organismos completamente sumergidos), 60, 120, 
240 y 360 min (Luquet \& Ansaldo 1997, Lagos \& Cáceres 2008). Los ejemplares fueron depositados en recipientes individuales de $250 \mathrm{~mL}$, que contenían arena esterilizada humedecida con agua de mar filtrada a $0,45 \mu \mathrm{m}$ (Greipsson \& El-Mayas 2000). Los recipientes fueron posteriormente colocados al interior de una cámara refrigerada por el tiempo de exposición definido, a la misma temperatura de aclimatación.

Para determinar el efecto de membranas respiratorias en el equilibrio ácido-base, se repitió el procedimiento anterior, esta vez con las membranas cubiertas con una capa de esmalte sintético $\left(\right.$ Revlon $\left.^{\circledR}\right)$, lo que imposibilita la función de intercambio gaseoso y no presenta efectos deletéreos sobre el organismo (Stillman 2000).

Al finalizar cada periodo experimental, se tomaron muestras de $1 \mathrm{~mL}$ de hemolinfa de cada individuo mediante punción en las quelas con jeringas de tuberculina. Inmediatamente después de su extracción las muestras fueron procesadas para medir los siguientes parámetros: $\mathrm{pH}$ con un pHímetro HANNA ${ }^{\circledR}$ modelo 1332; concentración de $\mathrm{HCO}_{3}{ }^{-}$, según el método de Seagal modificado para la determinación titrimétrica de $\mathrm{HCO}_{3}^{-}$(Henry et al. 1980); presión parcial de $\mathrm{CO}_{2}\left(\mathrm{PCO}_{2}\right)$ mediante la ecuación de Henderson \& Hasselbalch (Henry et al. 1981, Waldron et al. 1986, Byrne \& McMahon 1991, Varley \& Greenaway 1992), $\mathrm{pK}=\mathrm{pH}-\ln \left(\left[\mathrm{HCO}_{3}{ }^{-}\right] / \alpha \mathrm{CO}_{2} \times \mathrm{PCO}_{2}\right)$. Se utilizaron los valores $\mathrm{HCO}_{3}^{-}$y $\mathrm{pH}$ obtenidos en los experimentos anteriores y los valores de $\alpha \mathrm{CO}_{2}=0,0409$ y $\mathrm{pK}=6,073$ (Henry et al. 1981, Waldron et al. 1986).

Las relaciones entre área branquial, área de las membranas respiratorias y tamaño corporal fueron evaluadas mediante análisis de regresión lineal, las diferencias entre especies de pendientes e interceptos fueron evaluadas mediante una prueba de $t$ de Student (Zar 1996).

Los efectos de la funcionalidad de las membranas respiratorias en la exposición aérea entre especies sobre las variables medidas en la hemolinfa fueron evaluadas mediante ANDEVA de tres vías, utilizando factores fijos de especie, funcionalidad de las membranas y tiempo, seguido de una prueba a posteriori de Tukey. La homogeneidad de varianza se evaluó mediante la prueba de Levene y posteriormente los datos no homogéneos fueron transformados a $\log _{10}(\mathrm{x})$ (Zar 1996). Los valores se presentaron como promedio y su respectivo error estándar $(\mathrm{x} \pm \mathrm{ee})$.

\section{Resultados}

\section{Área de estructuras respiratorias}

La posición y número de las branquias en las dos especies fue similar, cada cámara branquial posee 14 branquias, de ellas dos branquias se encuentran asociadas al tercer maxilípedo, dos branquias al primer, tres al segundo, tres al tercer y tres al cuarto apéndice locomotor, y finalmente, una branquia al quinto apéndice locomotor vestigial.

El área branquial en ambas especies aumentó significativamente con el tamaño corporal $\left(\mathrm{r}^{2}=0,73, \mathrm{~N}=\right.$ 10 para Petrolisthes violaceus y $\mathrm{r}^{2}=0,63, \mathrm{~N}=10$ para P. laevigatus) (Fig. 1a) y no se encontraron diferencias significativas en pendientes ni en interceptos entre ambas especies (prueba t-Student, $\mathrm{t}=0,51, P=0,60 \mathrm{y} \mathrm{t}=0,63, P$ $=0,61$ respectivamente). El área branquial peso-específico

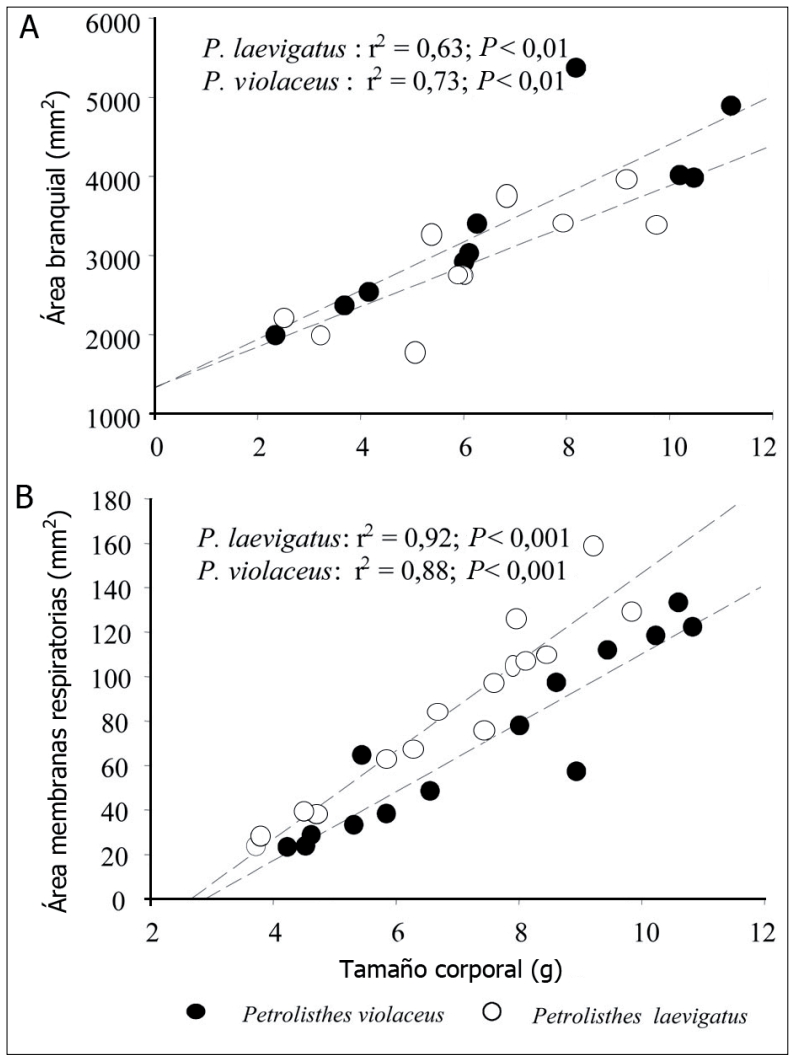

Figura 1. Relación entre área de estructuras respiratorias $\left(\mathrm{mm}^{2}\right)$ y masa corporal (g) en Petrolisthes laevigatus y $P$. violaceus. A) Área superficial branquial y B) Área de membranas respiratorias de los apéndices locomotores / Relationship between surface of respiratory structures $\left(\mathrm{mm}^{2}\right)$ and body weight $(\mathrm{g})$ in Petrolisthes laevigatus and $P$. violaceus. A) Surface gill area and B) Respiratory membranes area of walking legs 
fue similar en ambas especies: 509,73 $\pm 73,69 \mathrm{~mm}^{2} \mathrm{~g}^{-1}$ para P. laevigatus y $545,71 \pm 141,30 \mathrm{~mm}^{2} \mathrm{~g}^{-1}$ para $P$. violaceus (ANDEVA, $\mathrm{F}_{(1,18)}=0,28, P=0,60$ ) (Tabla 1).

Por otra parte, el área de las membranas respiratorias de los apéndices locomotores aumentó significativamente con el tamaño corporal en ambas especies $\left(\mathrm{r}^{2}=0,88, \mathrm{~N}=\right.$ 15 para $P$. violaceus y $\mathrm{r}^{2}=0,91, \mathrm{~N}=15$ para $P$. laevigatus) (Fig. 1b), existiendo diferencias tanto entre las pendientes de ambas relaciones como en los interceptos (prueba $\mathrm{t}$-Student, $\mathrm{t}=2,74, P<0,05$ para pendientes; $\mathrm{t}=2,34, P<$ 0,05 para interceptos).

\section{Equilibrio ÁCIDO-BASE}

Para el desarrollo de la parte experimental de este trabajo fueron seleccionados 150 individuos de cada especie de similares tamaños corporales (ANDEVA: $\mathrm{F}_{(1,298)}=1,05$; $P=0,31)$.

\section{pH}

Petrolisthes laevigatus tuvo un $\mathrm{pH}$ hemolinfático significativamente más básico $(7,89 \pm 0,02)$ que $P$. violaceus $(7,76 \pm 0,01)$ (ANDEVA, $\mathrm{F}_{(1,280)}=65,97, P<$ $0,01)$ (Tabla 1, Fig. 2a). También se observó un efecto de funcionalidad de las membranas sobre el $\mathrm{pH}$, con un mayor valor en los organismos con las membranas respiratorias funcionales comparado con los organismos con las membranas respiratorias obstruidas (ANDEVA, $\left.\mathrm{F}_{(1,280)}=79,00 P<0,01\right)$. El tiempo de exposición aérea igualmente afectó el $\mathrm{pH}$ de la hemolinfa, los mayores valores se midieron al comienzo del periodo experimental, $7,94 \pm 0,02$, y los mayores valores a los $360 \mathrm{~min}$ de exposición aérea, 7,73 $\pm 0,03$ (ANDEVA, $\mathrm{F}_{(4,280)}=$ 25,16, $P<0,01)$. Además, se observó una interacción significativa entre las variables especie y tiempo, siendo $P$. laevigatus a los 60 min de exposición aérea la especie con el $\mathrm{pH}$ más alto $(8,02 \pm 0,01)$, mientras que el $\mathrm{pH}$ más bajo fue medido en $P$. violaceus a los $360 \min (7,68 \pm 0,04)$ (Fig. 2a) (ANDEVA, $\left.\mathrm{F}_{(4,280)}=4,16 ; P<0,01\right)$ (Tukey $a$ posteriori $P<0,05)$. También se observó una interacción entre tiempo de emersión y obstrucción de las membranas respiratorias, siendo menores los valores de $\mathrm{pH}$ en los individuos con las membranas respiratorias obturadas a los 360 min de emersión (Fig. 2a) (ANDEVA, $\mathrm{F}_{(4,280)}=8,44$; $P<0,01$ ) (Tukey a posteriori $P<0,05$ ). La interacción entre las variables especie, tiempo de exposición aérea y funcionalidad de las membranas respiratorias también influye significativamente en el $\mathrm{pH}$ de la hemolinfa (ANDEVA: $\mathrm{F}_{(4,280)}=4.57 ; P<0,001$ ), observado claramente en $P$. laevigatus cuando a 60 min de exposición

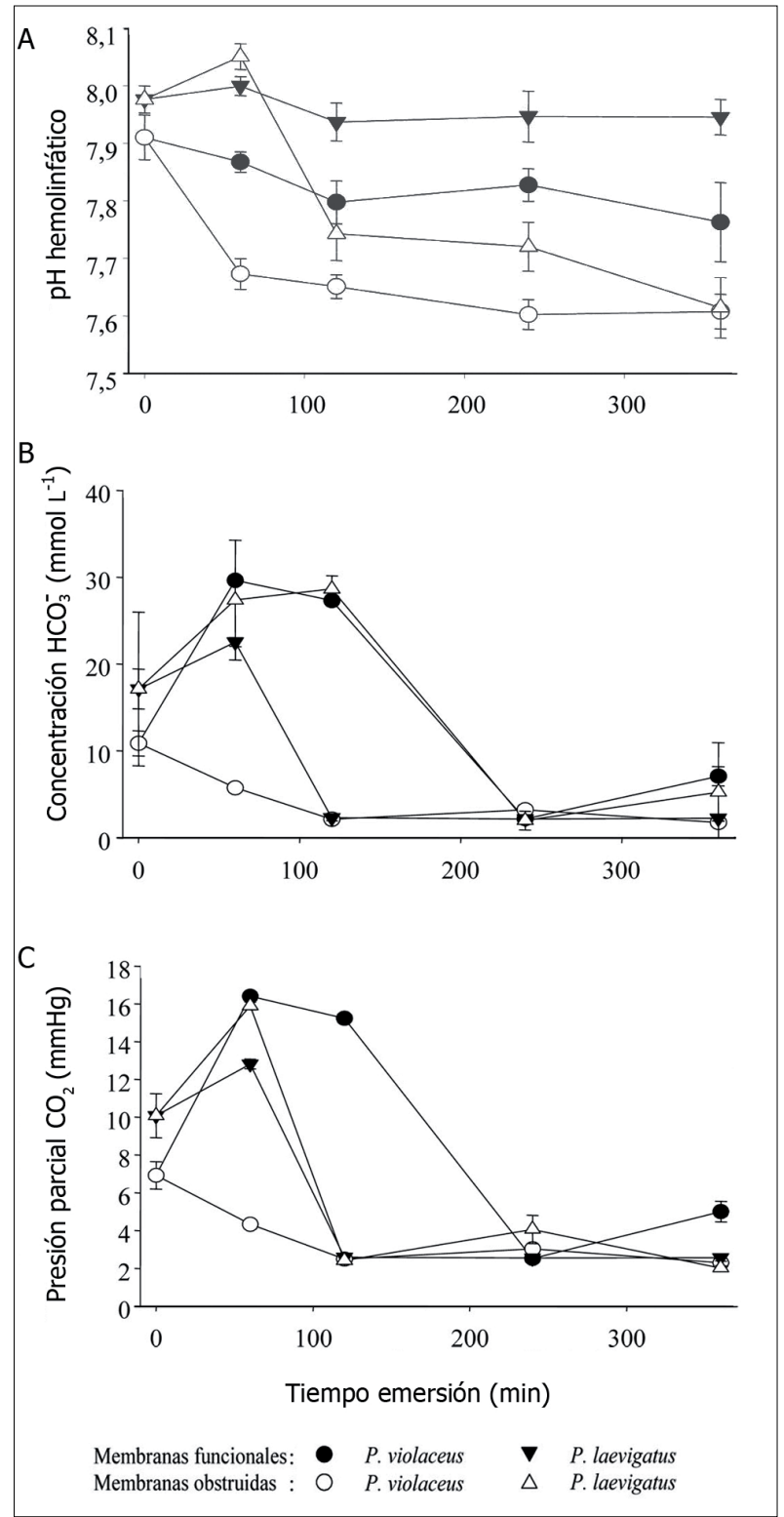

Figura 2. Valores promedios de A) variaciones de $\mathrm{pH}$ hemolinfático, B) concentración hemolinfática de $\mathrm{HCO}_{3}^{-}$y C) Presión parcial en la hemolinfa de $\mathrm{CO}_{2}$ en Petrolisthes laevigatus y $P$. violaceus bajo dos tratamientos: membranas respiratorias de los apéndices locomotores cubiertas y descubiertas a distintos tiempos de exposición aérea. Líneas verticales representan desviaciones estándares / Average A) variations in hemolymphatic $\mathrm{pH}, \mathrm{B}) \mathrm{HCO}_{3}^{-}$ hemolymphatic concentrations and C) Hemolymphatic $\mathrm{CO}_{2}$ partial pressure, in Petrolisthes laevigatus and $P$. violaceus under two treatments: respiratory membranes of walking legs covered and uncovered over several times of aerial exposure. Vertical lines represent standard deviations

aérea, con las membranas respiratorias obstruidas, fue el grupo con un mayor valor de $\mathrm{pH}(8,05 \pm 0,02)$, mientras que $P$. violaceus, también con las membranas obstruidas, 
Tabla 1. Resumen de los resultados de las ANDEVAs de tres vías para la relación entre las variables hemolinfáticas: a) Área branquial peso específico (ABPE), b) $\mathrm{pH}$, c) concentración de $\mathrm{HCO}_{3}^{-}$y d) $\mathrm{PCO}_{2}$; en relación al tiempo de exposición aérea y tratamiento membranas cubiertas y descubiertas de Petrolisthes laevigatus y $P$. violaceus $(G L=$ Grados de libertad, $C M=$ Cuadrados medios, $P=$ Probabilidad) / Three way ANOVA summary about hemolymphatic variables: a) Weight-specific gill area (ABPE), b) $\mathrm{pH}$, c) $\mathrm{HCO}_{3}{ }^{-}$concentration and d) $\mathrm{PCO}_{2}$ : in relationship with time of aerial exposition, and treatment, cover and uncovered membranes of Petrolisthes laevigatus and $P$. violaceus (GL= Freedom degrees, $\mathrm{CM}=$ Mean square, $\mathrm{P}=$ Probability value)

\begin{tabular}{|c|c|c|c|c|c|}
\hline & & $\mathrm{GL}$ & $\mathrm{CM}$ & $\mathrm{F}$ & $P$ \\
\hline \multirow[t]{2}{*}{ a) $\mathrm{ABPE}$} & Especie & 1 & 6471,12 & 0,28 & 0,59 \\
\hline & Error & 18 & 22334,63 & & \\
\hline \multirow[t]{8}{*}{ b) $\mathrm{pH}$} & Especie (1) & 1 & 1,27 & 65,97 & $<0,01$ \\
\hline & Tratamiento (2) & 1 & 1,52 & 79,00 & $<0,01$ \\
\hline & Tiempo (3) & 4 & 0,48 & 25,16 & $<0,01$ \\
\hline & $1 \times 2$ & 1 & 0,00 & 0,02 & 0,89 \\
\hline & $1 \times 3$ & 4 & 0,08 & 4,16 & $<0,01$ \\
\hline & $2 \times 3$ & 4 & 0,16 & 8,44 & $<0,01$ \\
\hline & $1 \times 2 \times 3$ & 4 & 0,09 & 4,57 & $<0,01$ \\
\hline & Error & 280 & 0,02 & & \\
\hline \multirow[t]{8}{*}{ c) $\mathrm{HCO}_{3}^{-}$} & Especie (1) & 1 & 304,64 & 18,32 & $<0,01$ \\
\hline & Tratamiento (2) & 1 & 3275,92 & 197,04 & $<0,01$ \\
\hline & Tiempo (3) & 4 & 2984,03 & 179,48 & $<0,01$ \\
\hline & $1 \times 2$ & 1 & 1243,39 & 74,79 & $<0,01$ \\
\hline & $1 \times 3$ & 4 & 1393,91 & 83,84 & $<0,01$ \\
\hline & $2 \times 3$ & 4 & 377,96 & 22,73 & $<0,01$ \\
\hline & $1 \times 2 \times 3$ & 4 & 1439,67 & 86,59 & $<0,01$ \\
\hline & Error & 280 & 16,63 & & \\
\hline \multirow[t]{8}{*}{ d) $\mathrm{PCO}_{2}$} & Especie (1) & 1 & 253984,80 & 133,19 & $<0,01$ \\
\hline & Tratamiento (2) & 1 & 300161,54 & 157,40 & $<0,01$ \\
\hline & Tiempo (3) & 4 & 63656,33 & 33,38 & $<0,01$ \\
\hline & $1 \times 2$ & 1 & 99634,72 & 52,25 & $<0,01$ \\
\hline & $1 \times 3$ & 4 & 23757,13 & 12,46 & $<0,01$ \\
\hline & $2 \times 3$ & 4 & 89972,13 & 47,18 & $<0,01$ \\
\hline & $1 \times 2 \times 3$ & 4 & 1906,96 & 73,83 & $<0,01$ \\
\hline & Error & 280 & 4,26 & & \\
\hline
\end{tabular}

presentó valores significativamente mas bajos de $\mathrm{pH}$ a los $240 \min (7,60 \pm 0,02)$ y $360 \min (7,60 \pm 0,03)$ de exposición aérea (Tukey a posteriori $P<0,05)$ (Tabla 1 ).

\section{$\mathrm{HCO}_{3}^{-}$}

En esta variable el resultado fue un efecto significativo de la especie, del tratamiento obstrucción de las membranas respiratorias y del tiempo de exposición aérea (ANDEVA: $\mathrm{F}_{(1,280)}=18,32 ; P<0,01$ para especie; $\mathrm{F}_{(1,280)}=197,04$; $P<0,01$ para tratamiento y $\mathrm{F}_{(4,280)}=179,04 ; P<0,01$ para tiempo) (Fig. 2b, Tabla 1). Se observó un efecto significativo de la interacción entre especie y funcionalidad de las membranas respiratorias, siendo $P$. violaceus, con las membranas respiratorias obturadas, el grupo que presentó la menor concentración de $\mathrm{HCO}_{3}^{-}, 4,75 \pm 0,50 \mathrm{mmol} \mathrm{L}^{-1}$ (ANDEVA: $\mathrm{F}_{(1,280)}=74,79 ; P<0,01$ ) (Tukey a posteriori $P<0,05)$. Al mismo tiempo hubo una interacción entre el factor especie y tiempo a los 60 min de exposición aérea, ya que con las membranas obturadas, P. laevigatus presentó la mayor concentración de $\mathrm{HCO}_{3}^{-}, 28,6 \pm 0,40$ 
mmol L$^{-1}$ (Fig. 2b) (ANDEVA F ${ }_{(4,280)}=83,84 ; P<0,01$ ) (Tukey a posteriori $P<0,05$ ). Los organismos con las membranas obstruidas de ambas especies presentaron las menores concentraciones de $\mathrm{HCO}_{3}{ }^{-}$entre los 120 y 360 min de exposición aérea (ANDEVA: $\mathrm{F}_{(4,280)}=22,73 ; P$ $<0,01$ ) (Tukey a posteriori $P<0,05$ ) (Tabla 1, Fig. 2b). Finalmente se observó una interacción entre los tres factores experimentales, especie, tratamiento y tiempo, siendo los menores valores de concentración a los $60 \mathrm{~min}$ de exposición aérea, en los grupos de $P$. violaceus con las membranas respiratorias funcionales y en $P$. laevigatus con las membranas obstruidas (ANDEVA: $\mathrm{F}_{(4,280)}=86,59$; $P<0,01$ ) (Tukey a posteriori $P<0,05$ )

\section{$\mathrm{PCO}_{2}$}

En la $\mathrm{PCO}_{2}$ se encontró un efecto significativo de la especie, del tratamiento, obstrucción de las membranas respiratorias y del tiempo de exposición aérea (ANDEVA: $\mathrm{F}_{(1,280)}=$ 113,$19 ; P<0,01$ para especie; $\mathrm{F}_{(1,280)}=157,40 ; P<0,01$ para tratamiento y $\mathrm{F}_{(4,280)}=33,38 ; P<0,01$ para tiempo) (Tabla 1, Fig. 2c). Se observó una interacción significativa entre especie y obstrucción de las membranas respiratorias en $P$. violaceus, a los 360 min de exposición aérea con las membranas obstruidas y fue el grupo que presentó el menor valor con 2,28 $\pm 0,25 \mathrm{mmHg}$ (ANDEVA: $\mathrm{F}_{(1,280)}$ $=52,25 ; P<0,001)$ (Tukey a posteriori $P<0,05)$ (Tabla 1). Además, se observó una interacción significativa entre tratamiento de las membranas respiratorias con el tiempo ya que a los 60 min de exposición aérea se encontraron valores de $\mathrm{PCO}_{2}$ significativamente mayores que en los otros grupos experimentales (ANDEVA: $\mathrm{F}_{(4,280)}=47,18$; $P<0,01$ ) (Tukey a posteriori $P<0,05$ ) (Fig. 2c). También existió una interacción significativa entre especie y tiempo, siendo $P$. laevigatus con las membranas respiratorias obstruidas a los 360 min de exposición aérea, el grupo con los menores valores de $\mathrm{PCO}_{2}, 2,00 \pm 0,12 \mathrm{mmHg}$ (ANDEVA: $\mathrm{F}_{(4,280)}=12,46 P<0,01$ ) (Tukey a posteriori $P<0,05)$. En esta variable, la interacción entre los tres factores experimentales fue significativa, siendo menores los valores de $\mathrm{PCO}_{2}$ medidos a los 360 min de exposición aérea en los grupos de $P$. violaceus y $P$. laevigatus con las membranas respiratorias obstruidas y los mayores valores se observaron a los 60 min en los grupos de $P$. laevigatus con las membranas obstruidas con $15,89 \pm 0,20 \mathrm{mmHg}$ y de $P$. violaceus con las membrana funcionales con $16,40 \pm$ 0,06 mmHg (ANDEVA: $\mathrm{F}_{(4,280)}=73,83 ; P<0,01$ ) (Tukey a posteriori $P<0,05)$.

\section{Discusión}

En este estudio no se encontraron diferencias en el área de la superficie de las branquias y la estructura primaria de intercambio gaseoso en inmersión entre Petrolisthes violaceus y $P$. laevigatus. En cambio, el área de las membranas respiratorias de los apéndices locomotores fue significativamente diferente entre ambas especies y estas membranas cumplen un rol diferencial en la regulación del equilibrio acido base durante la exposición aérea en ambas especies.

Ambas especies de Petrolisthes presentaron un área superficial branquial baja, cercana a $500 \mathrm{~mm}^{2} \mathrm{~g}^{-1}$ (Tabla 2). Este valor se encuentra dentro del rango de los organismos intermareales (Gray 1957, Henry 1994) y en una posición intermedia, que presentan valores más altos que los encontrados en crustáceos de habitats terrestres, los cuales utilizan respiración bimodal y pulmonar (Mangum 1994, Halperin et al. 2000) y a su vez menores que el área branquial de crustáceos exclusivamente acuáticos (Gray 1957) (Tabla 2).

La existencia de membranas respiratorias accesorias ubicadas en los apéndices locomotores ha sido descrita en dos grupos de crustáceos filogenéticamente lejanos, los cangrejos fantasmas braquiuros Scopimera y Dotilla de Australia (Maitland 1986) y los porcelánidos anomuros Petrolisthes (Stillman \& Somero, 1996). En ambos grupos, estas membranas cumplirían un rol en la respiración aérea durante periodos de alta demanda metabólica como son los periodos de emersión (Stillman 2000). En este estudio, la mayor área de membranas respiratorias se observó en $P$. laevigatus comparado con las de P. violaceus (Fig. 2b), por lo que le facilitaría a esta especie un mayor intercambio gaseoso en periodos de emersión y mejor adaptación de la franja superior del intermareal.

En relación al $\mathrm{pH}$ de la hemolinfa, en los primeros 60 a 120 min de exposición, se observó la mayor variación en ambas especies, siendo significativamente mayor el efecto de la exposición aérea en los tratamientos con membranas obstruidas. Posteriormente se observó una estabilización del $\mathrm{pH}$, pero manteniéndose los niveles menores en los grupos experimentales con membranas no funcionales. Un comportamiento similar se ha observado en otras especies como Chasmagnathus granulata (Luquet \& Ansaldo 1997), cuyas mayores variaciones de $\mathrm{pH}$ se observaron dentro de la primera hora de exposición aérea. En general, la regulación de $\mathrm{pH}$ depende del balance entre iones, en este aspecto participan dos mecanismos; en crustáceos 
Tabla 2. Rangos comparativos del área superficial branquial peso específico de cada grupo representativo de transición evolutiva desde habitats acuáticos a terrestres en crustáceos decápodos / Comparative ranges of weight-specific gill surface areas of each representative group related to evolutionary transition from the aquatic to terrestrial habitats in decapod crustaceans

\begin{tabular}{lcl}
\hline Tipo de respiración & Área peso especifico $\left(\mathrm{mm}^{2} \mathrm{~g}^{-1}\right)$ & \multicolumn{1}{c}{ Fuente } \\
\hline Acuático & $900-1400$ & Henry 1994, Gray 1957 \\
Intermareal & $500-900$ & Henry 1994, Gray 1957 \\
$\quad$ P. laevigatus & 510 & Este estudio \\
$\quad$ P. violaceus & 546 & Este estudio \\
Bimodal & $35-325$ & Henry 1994, Gray 1957 \\
Terrestre & $12-500$ & Henry 1994, Gray 1957 \\
\hline
\end{tabular}

acuáticos se regularía mediante intercambio iónico por medio de las branquias, mientras que en crustáceos terrestres y semiterrestres sería a través de la disolución de $\mathrm{CaCO}_{3}$ del exoesqueleto, lo que genera $\mathrm{Ca}^{2+}$ y $\mathrm{HCO}_{3}{ }_{3}$ (Henry et al. 1981, Truchot 1990, Luquet \& Ansaldo 1997, Lagos \& Cáceres 2008). La diferencia del pH entre los grupos con las membranas libres y obstruidas fue mayor en P. laevigatus, por lo cual indica un mayor rol de estas estructuras en la regulación del equilibrio ácido base.

Uno de los principales mecanismos que utilizan los animales para regular el $\mathrm{pH}$ de sus fluidos extracelulares sería mediante el uso de tampones orgánicos o inorgánicos (Henry \& Wheatly 1992). Los tampones más importantes utilizados en la regulación del $\mathrm{pH}$ en la hemolinfa es el sistema $\mathrm{CO}_{2} / \mathrm{HCO}_{3}^{-}$(Henry et al. 1981, Henry \& Wheatly 1992), el cual en condiciones normales se facilitaría por un intercambio activo de iones $\mathrm{Na}^{+}$y $\mathrm{Cl}^{-}$a nivel branquial. En exposición aérea, este intercambio no se realiza, lo cual promueve la acumulación de $\mathrm{CO}_{2}$ y la acidificación de la hemolinfa (Luquet \& Ansaldo 1997, Henry \& Cameron 1982, Henry et al. 1981, Truchot 1990, Wheatly \& Henry 1992).

Según Randall (2002) la disminución en el pH, combinado con un alto $\mathrm{PCO}_{2}$ y un aumento en la concentración de $\mathrm{HCO}_{3}{ }^{-}$son indicadores de una acidosis respiratoria. En el presente trabajo se observó esta combinación, tanto para $P$. violaceus como para $P$. laevigatus. Este efecto fue evidente en los organismos con las membranas respiratorias obstruidas en los primeros minutos hasta las $4 \mathrm{~h}$ de exposición aérea, manteniéndose constante posteriormente, lo que indicaría que estos organismos realizan un importante intercambio gaseoso a través de estas estructuras, particularmente en las primeras horas de exposición aérea.
Las respuestas en las concentraciones de $\mathrm{HCO}_{3}^{-}$y $\mathrm{PCO}_{2}$ en ambas especies tuvieron un comportamiento similar, es decir, aumentó los primeros $60 \mathrm{~min}$ con un posterior descenso hasta los $240 \mathrm{~min}$, esto concuerda con lo observado en la disminución del $\mathrm{pH}$ en los organismos con las membranas respiratorias funcionales. Esta respuesta es similar a lo descrito para otras especies como Callinectes sapidus cuya compensación de una acidosis fue acompañada por captación de $\mathrm{HCO}_{3}{ }^{-}$(Henry \& Wheatly 1992, Whiteley et al. 2001). El aumento de la concentración hemolinfática de $\mathrm{HCO}_{3}{ }^{-}$, en el comienzo de la exposición aérea, probablemente estaría facilitada por dos factores, uno sería la retención de agua en la cámara branquial, lo que permitiría el intercambio iónico (Halperin et al. 2000), o bien, por la capacidad de disolver el $\mathrm{CaCO}_{3}{ }^{-}$ del exoesqueleto en $\mathrm{Ca}^{2+}+\mathrm{HCO}_{3}{ }^{-}$sugerido en literatura (Henry et al. 1981) y cuantificado en P. laevigatus (Lagos \& Cáceres 2008).

Los resultados sugieren que estos crustáceos, estarían usando activamente las membranas de los apéndices locomotores como órgano respiratorio en condiciones de emersión, en donde P. laevigatus con membranas respiratorias anexas de mayor tamaño y mayor superficie para el intercambio de gases respiratorios, tendría una ventaja comparativa con respecto a $P$. violaceus, permitiéndole habitar en la zona alta del intermareal con periodos de emersión más prolongados.

\section{Agradecimientos}

El presente escrito formó en parte la tesis de grado de M. A. Vargas, para optar al grado académico de Licenciado en Ciencias, con mención de Biología Marina y al título profesional de Biólogo Marino de la Universidad Católica de la Ssma. Concepción. Los autores agradecen a los 
evaluadores anónimos por sus sugerencias y comentarios que mejoraron el trabajo sustantivamente.

\section{LITERATURA CITADA}

Astall CA, SJ Anderson \& AC Taylor. 1997. Comparative studies of the branchial morphology, gill area and gill ultrastructure of some thalassinidean mud-shrimps (Crustacea: Decapoda: Thalassinidea). Journal of Zoology 241: 665-688.

Byrne RA \& BR Mc Mahon. 1991. Acid-base and ionic regulation, during and following emersion, in the freshwater Bivalve, Anodonta grandis simpsoniana (Bivalvia: Unionidae). The Biological Bulletin 181: 289-297.

Castillo-Blasco CA, ME Lagos \& CW Cáceres. 2009. Osmoregulación y equilibrio ácido-base en el crustáceo Neotrypaea uncinata (Milne Edwards, 1837) (Decapoda: Thalassinidae): consecuencias del parasitismo por Ionella agassizi (Bonnier, 1900) (Isopoda: Bopyridae). Revista de Biología Marina y Oceanografía 44(3): 715-724.

Chen JC \& PG Chia. 1997. Osmotic and ionic concentrations of Scylla serrata (Porskal) subjected to different salinity levels. Comparative Biochemistry and Physiology A 117: 239-244.

Farrelly C \& P Greenaway. 1987. The morphology and vasculature of the lungs and gills of the soldier crabs, Mictyris longicarpus. Journal of Morphology 193: 282304.

Farrelly CA \& P Greenaway. 1994. Gas exchange trough the lungs and gills in air-breathing crabs. The Journal of Experimental Biology 187: 113-130.

Gray IE. 1957. A comparative study of gill area of crabs. The Biological Bulletin 112: 34-42.

Greenaway P. 1984. The relative importance of the gills and lungs in the gas exchange of amphibious crabs of the genus Holthuisana. Australian Journal of Zoology 32: 1-6.

Greenaway P. 2003. Terrestrial adaptation in the Anomura (Crustacea: Decapoda). Memoirs of Museum Victoria 60(1): 13-26.

Greipsson S \& H El-Maya. 2000. Arbuscular mycorrhizae of Leymus arenarius on coastal sands and reclamation sites in Iceland and response to inoculation. Restoration Ecology 8: $144-150$.

Halperin J, M Ansaldo, GN Pellerano \& CM Luquet. 2000. Bimodal breathing in the estuarine crab Chasmagnathus granulatus Dana 1851, physiological and morphological studies. Comparative Biochemistry and Physiology B 126: 341-349.

Hawkins AJS \& MB Jones. 1982. Gill area and ventilation in two mud crabs, Helice crassa Dana (Grapsidae) and
Macrophthalmus hirtipes (Jacquinot) (Ocypodidae), in relation to habitat. Journal of Experimental Marine Biology and Ecology 60: 103-118.

Hawkins AJS, MB Jones \& ID Marsden. 1982. Aerial and aquatic respiration in two mud crabs, Helice crassa Dana (Grapsidae) and Macrophthalmus hirtipes (Jacquinot) (Ocypodidae), in relation to habitat. Comparative Biochemistry and Physiology A 73: 341-347.

Henry R, D Cannon \& J Winkelman. 1980. Química clínica, bases y técnicas. Tomo 1, 819 pp. Editorial Jims, Barcelona.

Henry RP. 1994. Morphological, behavioral, and physiological characterization of bimodal breathing crustaceans. American Zoologist 34: 205-215.

Henry RP \& JN Cameron. 1982. Acid base balance in Callinectes sapidus during acclimation from high to low salinity. Journal of Experimental Biology 101: 255-264.

Henry RP \& MG Wheatly. 1992. Interaction of respiration, ion regulation, and acid-base balance in the everyday life of aquatic crustaceans. American Zoologist 32: 407-416.

Henry RP, GA Kormanik, NJ Smatresk \& JN Cameron. 1981. The role of $\mathrm{CaCO}_{3}$ dissolution as a source of $\mathrm{HCO}_{3}$ for the buffering of hypercapnic acidosis in aquatic and terrestrial decapod crustaceans. Journal of Experimental Biology 94: 269-274.

Innes AJ, ME Forster, MB Jones, ID Marsden \& HH Taylor. 1986. Bimodal respiration, water balance and acidbase regulation in a high-shore crab, Cyclograpsus lavauxi. Journal of Experimental Marine Biology and Ecology 100: 127-145.

Lagos ME \& CW Cáceres. 2008. Como afecta la exposición aérea el equilibrio ácido base de organismos móviles del intermareal: Petrolisthes laevigatus (Guérin, 1835) (Decapoda: Porcellanidae) como caso de estudio. Revista de Biología Marina y Oceanografía 43: 591-598.

Lucu C \& M Devescovi. 1999. Osmoregulation and branchial $\mathrm{Na}^{+}, \mathrm{K}^{+}$-ATPase in the lobster Homarus gammarus acclimated to dilute seawater. Journal of Experimental Marine Biology and Ecology 234: 291-304.

Luquet CM \& M Ansaldo. 1997. Acid-base and ionic regulation during emersion in the estuarine intertidal crab Chasmagnathus granulata Dana (Decapoda, Grapsidae). Comparative Biochemistry and Physiology A 117: 407410.

Luquet CM, CO Cervino, M Ansaldo, V Carrera, S Kocnur \& RE Dezi. 1998. Physiological response to emersion in the amphibius crab Chasmagnathus granulata Dana (Decapoda Grapsidae); biochemical and ventilatory adaptations. Comparative Biochemistry and Physiology A 121: 385-393. 
Maitland DP. 1986. Crabs that breathe air with their legsScopimera and Dotilla. Nature 319: 493-495.

Maitland DP. 1990. Aerial respiration in the semaphore crab, Heloecius cordiforms, with or without branchial water. Comparative Biochemistry and Physiology A 95: 267-274.

Mangum CP. 1994. Multiple sites of gas exchange. American Zoologist 34: 184-193.

Onken H, M Tresquerres \& CM Luquet. 2003. Active $\mathrm{NaCl}$ absorption across posterior gills of hyperosmoregulating Chasmagnathus granulatus. The Journal of Experimental Biology 206: 1017-1023.

Randall D, W Burggren \& K French. 2002. Eckert, animal physiology: mechanisms and adaptations, 735 pp. W. H. Freeman Company, New York.

Stillman JH. 2000. Evolutionary history and adaptative significance of respiratory structures on the legs of intertidal Porcelain crabs, Genus Petrolisthes. Physiological and Biochemical Zoology 73(1): 86-96.

Stillman JH \& GN Somero. 1996. Adaptation to temperature stress and aerial exposure in congeneric species of intertidal porcelain crabs (genus Petrolisthes) correlation of physiology, biochemistry and morphology with vertical distribution. The Journal of Experimental Biology 199: 1845-1855.
Truchot JP. 1990. Respiratory and ionic regulation in invertebrates exposed to both water and air. Annual Review of Physiology 52: 61-74.

Varley D \& P Greenaway. 1992. The effect of emersion on hemolimph acid-base balance and oxygen levels in Silla serrata Forskal (Brachyura: Portunidae). Journal of Experimental Biology and Ecology 163: 1-12.

Viviani CA. 1969. Los Porcellanidae (Crustacea, Anomura) chilenos. Sonderdruck aus Beiträge zur Neotropischen Fauna 6(1): 39-56.

Waldron FM, HH Taylor \& ME Foster. 1986. Acid-base disturbances following exercise in a high-shore crab, Cyclograpsus lavauxi. New Zealand Journal of Marine and Freshwater Research 20: 479-487.

Wheatly MG \& RP Henry. 1992. Extracellular and intracellular acid-base regulation in crustaceans. Journal of Experimental Zoology 263: 127-142.

Whiteley NM, JL Scott, SJ Breeze \& L McCann. 2001. Effects of water salinity on acid-base balance in decapod crustaceans. The Journal of Experimental Biology 204: 1003-1001.

Zar JH. 1996. Biostatistical analysis, 662 pp. Prentice Hall, Englewood Cliffs.

Recibido el 26 de noviembre de 2009 y aceptado el 26 de abril de 2010 